\title{
The CCD and readout electronics for the OMC instrument on Integral
}

\author{
D. M. Walton ${ }^{1}$, P. D. Thomas ${ }^{1}$, J. L. Culhane ${ }^{1}$, B. Jordan ${ }^{2}$, A. Smith ${ }^{1}$, A. P. Dibbens ${ }^{1}$, and L. J. Bradley ${ }^{1}$ \\ ${ }^{1}$ Mullard Space Science Laboratory, University College London, Holmbury St Mary, Dorking, Surrey, RH5 6NT, UK \\ ${ }^{2}$ Dunsink Observatory, Castleknock, Dublin 15, Ireland
}

Received 14 July 2003 / Accepted 18 September 2003

\begin{abstract}
The Optical Monitoring Camera (OMC) on ESA's Integral gamma-ray astronomy satellite is devoted to optical wavelength observations simultaneously covering the same field-of-view as the gamma-ray and X-ray instruments. The OMC consists of a refracting telescope with a CCD as the imaging device in the focal plane. Here we describe the CCD and its associated readout electronics, in particular pointing out features of interest to users of the OMC instrument and its data.
\end{abstract}

Key words. instrumentation: photometers - CCDs

\section{Introduction}

ESA's Integral gamma-ray astonomy satellite was launched on 17th October 2002 from Baikonur using a Russian Proton rocket. On board are 4 instruments, described in this journal. The purpose of the mission is to study astrophysical objects which emit in the gamma-ray portion of the electromagnetic spectrum. In order to understand the physical processes producing these phenomena it is necessary to also make observations in other spectral regions. Hence Integral is also equipped with instruments to observe in the X-ray (JEM-X) and optical (Optical Monitoring Camera, OMC) regions. For the OMC, the requirement to simultaneously cover the same field-of-view (FOV) as the X-ray and gamma-ray instruments with relatively high spatial resolution led to the choice of a refracting telescope with a CCD as the imaging device in the focal plane. The OMC hardware consortium consists of INTA (Spain, PI institute), MSSL (UK), CSL (Belgium) and UCD/DIAS (Ireland). The OMC is described in Mas-Hesse et al. (2003), and the telescope optics in Mazy et al. (2003). Here we describe the CCD and its associated electronics.

\subsection{Requirements}

Key requirements for the OMC's performance are:

- to cover a $5^{\circ} \times 5^{\circ} \mathrm{FOV}$;

- to reach a visual magnitude limit of $\sim 18$ for a sequence of $10100 \mathrm{~s}$ integrations;

- a spatial resolution of $\sim 20^{\prime \prime}$ to avoid field crowding;

- a time resolution of a few seconds for timing studies and to maintain a high duty cycle without saturation from bright sources.

Send offprint requests to: D. M. Walton,

e-mail: dmw@mssl.ucl.ac.uk
Following assessment within the OMC hardware consortium and discussions with the CCD manufacturer (e2v technologies, then EEV), the e2v CCD47-20 CCD was chosen as the imager. This device has an active image area of $1024 \times 102413 \mu \mathrm{m}$ pixels, meeting the FOV and resolution requirements. A backilluminated version was chosen to maximise the quantum efficiency (QE). This CCD has a frame transfer architecture, which allows a $100 \%$ observing duty cycle and eliminates the need for a shutter.

\subsection{Overview of electronic design}

Factors which constrain the design of the electronics include

- the need to interface with a pre-defined Data Processing Electronics (DPE);

- the necessity of a high degree of autonomy in order to minimise loading of the DPE;

- the limited space available in the detector head.

The approach taken has been to maximise both the flexibility and independence of the electronics while minimising intervention from the DPE. The space in the detector head is sufficient only for level shifters for the CCD clock signals, bias supply filtering, and preamplifiers for the CCD video output. This led to the concept shown in Fig. 1, where the other functions are housed in a separate ReadOut Electronics (ROE) unit, situated between the DPE and the Focal Plane Array (FPA). The DPE controls various ROE properties, such as integration time, window co-ordinates, binning factors etc. using a combination of bi-level commands and a 32 KBaud Low-speed Serial Link (LSL). The clock sequence generator creates all the CCD clocking signals at $5 \mathrm{~V}$ logic levels. The FPA electronics (FPAe) filters the CCD bias supplies from the ROE and levelshifts the $5 \mathrm{~V}$ CCD clocking signals to $\sim 0$ to $12 \mathrm{~V}$. These biases 


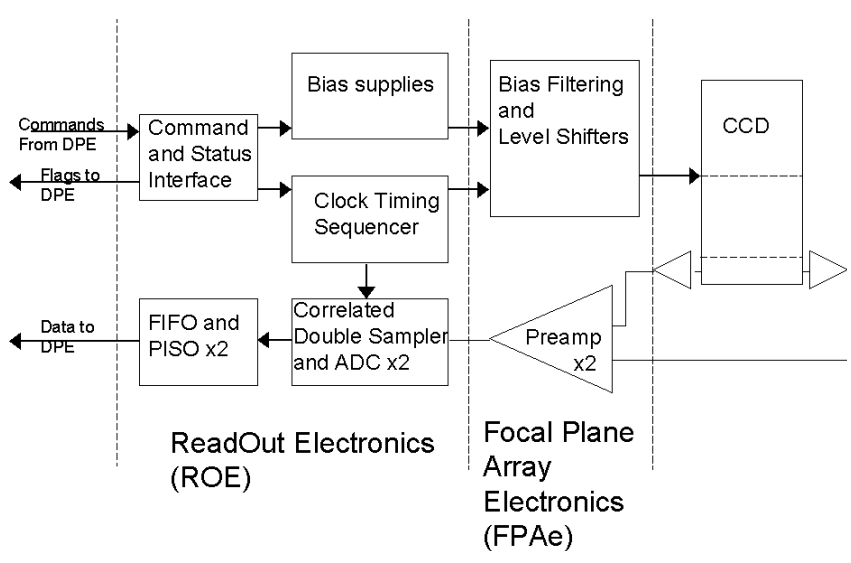

Fig. 1. Block diagram of OMC CCD and associated electronics.

and clocks drive the CCD. Each of the two CCD output nodes has an independent chain of amplifier, correlated double sampler (CDS), analogue to digital converter (ADC), FIFO buffer, PISO (parallel in serial out) and $5 \mathrm{MHz}$ high-speed serial link (HSL) to the DPE. The video signal from the CCD is amplified on the FPA card, giving a signal which can be transmitted down the $700 \mathrm{~mm}$ long cable to the ROE. The ROE contains the CDS, ADC, FIFO, PISO and HSL circuitry.

\section{Design and operation}

\subsection{CCD parameters}

The fundamental performance-determining factors for the OMC are set by the CCD. The CCD47-20 is a frame transfer CCD with an image area of $1056 \times 1028$ pixels of $13 \mu \mathrm{m} \times$ $13 \mu \mathrm{m}$ (the central $1024 \times 1024$ are the active area, the surrounding pixels are masked and can be used for dark reference and dark current measurement). The readout register is split in the middle with separate clock electrodes for each half and an output node at each end. By appropriate configuration of the $R \phi$ (readout register) clock signals, readout can be achieved to the left or right output port, or half in each direction. Simultaneous readout in both directions can be used to reduce the readout time by a factor 2 , alternatively the two output nodes can be used to give a degree of redundancy. All clocks are three phase. The two output ports are of low noise design, with a readout noise (using correlated double sampling) for the backilluminated version of $\sim 6 \mathrm{e}^{-} \mathrm{rms}$ at the OMC readout rate of $328 \mathrm{Kpix} \mathrm{s}^{-1}$. The OMC row shift time of $2.3 \mu$ s gives a frame transfer time of $\sim 2.4 \mathrm{~ms}$, so that integrations of a few seconds will have frame shift smear of $\sim 0.1 \%$. The CCD has a dump gate and drain running alongside the readout register: this facilitates flushing the CCD and dumping unwanted areas in a windowed imaging mode.

The telescope is designed to operate in the photometric $V$ band for comparison with standard astronomical catalogues, a $V$-band filter in the optical path defines the passband ( $\sim 500$ to $600 \mathrm{~nm}$ FWHM). The CCD is back-illuminated to maximise QE, with a standard anti-reflection coating to match the filter passband. Figure 2 shows QE as a function of wavelength for a $\mathrm{CCD}$ with this coating.

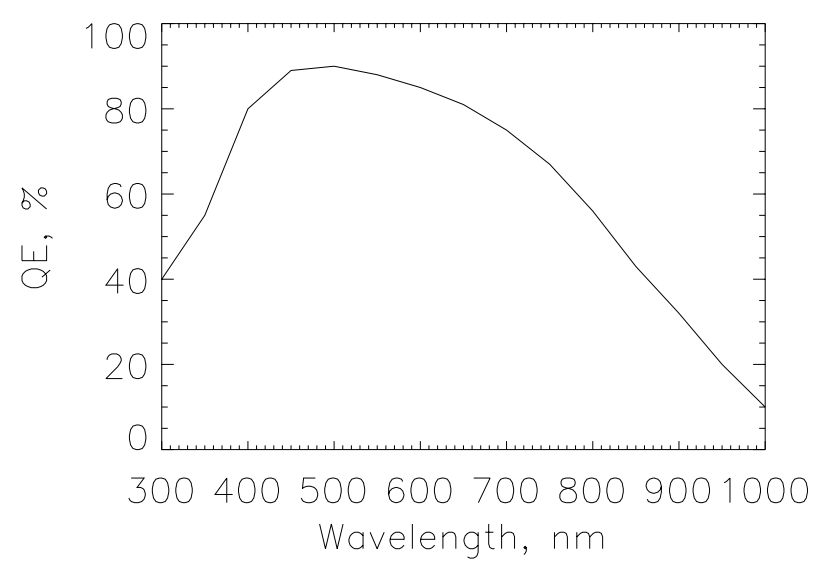

Fig. 2. $C C D Q E$ as a function of wavelength.

Two trade-offs to be made in selecting device type are as follows.

1. Inverted mode. An AIMO (Advanced Inverted Mode Operation) device has much lower dark current and hence eases cooling requirements. However, the penalty is a lower full-well capacity (and hence dynamic range) and greater dark signal non-uniformity (DSNU). It was decided to use a non-AIMO (or NIMO) device.

2. Anti-blooming. The trade-off here is that anti-blooming prevents the charge from bright stars from spreading up and down image columns, saturating these areas. However, anti-blooming also reduces full-well capacity. Given the relative infrequency of bright stars and the small area of the image affected, it was decided to use a CCD without anti-blooming.

The CCD is passively cooled to $\sim-80 \mathrm{C}$ for two reasons

- To reduce dark current to a sufficiently low level to allow long exposures. Dark current as a function of temperature for NIMO devices is given by

$I_{\mathrm{d}}=C T^{3} \exp [-6400 / T]$

where

$I_{\mathrm{d}}$ is the dark current

$C$ is a normalisation constant

$T$ is the temperature (Kelvin).

At $-80 \mathrm{C}$, the predicted dark current is $\sim 4 \times$ $10^{-2} \mathrm{e}^{-} \mathrm{pix}^{-1} \mathrm{~s}^{-1}$. In principle this allows exposures up to $\sim 10^{6} \mathrm{~s}$.

- To reduce the effects of radiation damage to acceptable levels.

At this temperature the normal ceramic dual-in-line IC package for this device is unsuitable as the focal plane flatness requirement would not be met. Therefore the silicon is mounted on a custom invar mounting disc, and connection is made using a flexi-track at each end of the chip wirebonded to the bond pads on the silicon, as shown in Fig. 3, which shows an engineering model of the CCD. 


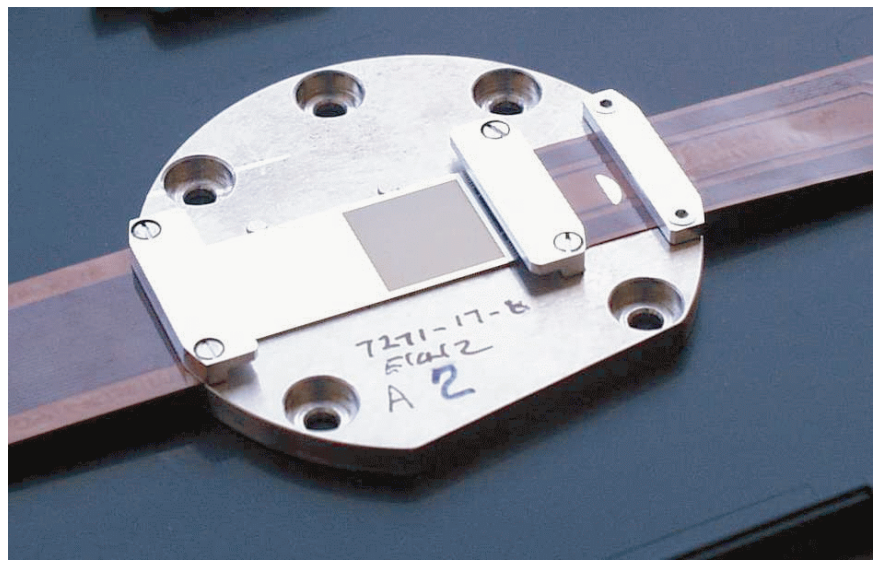

Fig. 3. Photograph of CCD with flexi-tracks on invar mount.

The other end of each flexi mates with connectors on the FPA electronics PCB. The flexi provides an electrical connection with reasonably low thermal conductivity between the $\mathrm{CCD}$ at $\sim-80 \mathrm{C}$ and the FPAe at $\sim 0 \mathrm{C}$. The FPAe has a thermostatic heater to prevent its temperature dropping below $-40 \mathrm{C}$ in order not to violate the temperature range of some of the components. Another factor in the design of the focal plane is contamination. The CCD at $-80 \mathrm{C}$ will act as a surface for molecular contamination to condense on. The FPA has been designed to allow heating of the CCD to $\sim 20 \mathrm{C}$ to re-evaporate these contaminants. Thus the $\mathrm{CCD}$, mounting and flexi had to be designed using low outgassing materials and be able to withstand temperature cycling over this range.

\subsection{Bias supplies and clock sequencer}

Bias supplies are derived from a $36 \mathrm{~V}$ supply generated in the PCE (Power Conditioning Electronics, supplied by INTA) and are generated using stable analogue control circuitry. Two of the supplies, $V_{\mathrm{SS}}$ (the CCD substrate bias supply) and $V_{\mathrm{RD}}$ (the output stage reset drain), can be controlled in order to allow for in-orbit flat-band shifts caused by ionising radiation (see below). This is achieved by the DPE loading registers in the ROE using the LSL. Each of these registers controls a DAC, the output of which controls the supply.

The clock sequencer consists of a card of HCMOS logic circuitry which generates all the CCD clocking waveforms and CDS timing waveforms. State machines generate the individual triplets for the three-phase clocking, while counters enable and disable the state machines in an appropriate sequence. The counters are loaded with default values from the ROE PROM at boot-up, but can be changed by the DPE, again loading ROE registers using the LSL. For example, the default integration time is $10 \mathrm{~s}$, but the DPE can control this in the range $25 \mathrm{~ms}$ to $819.2 \mathrm{~s}$. In principle, even longer integration times can be achieved by the DPE loading registers and setting and monitoring flags appropriately. Other parameters which can be adjusted include window size and position and binning factor. Lesser used parameters can also be changed, such as the frame transfer count. This makes the readout highly flexible: for example a Time Delay and Integration (TDI) mode could be effected; and for other applications of the ROE, different CCD types can be accommodated. The master clock for the sequencer is the $5 \mathrm{MHz}$ HSL clock. This gives a pixel period of $3.05 \mu$ s and a readout time for a full image of $\sim 3.5 \mathrm{~s}$.

\subsection{Analogue signal chain, $A D C$ and DPE data interface}

The signal from each output node of the CCD is amplified in the FPAe first by a low noise FET, then a low noise operational amplifier (op-amp), then a line driver op-amp. This combination amplifies the CCD output by $\times 16$, raising the $\sim 0.5 \mathrm{~V}$ maximum signal to a sufficiently high level to allow transmission from the FPA to the ROE. In the ROE the signal is processed by the CDS, is amplified by another factor 1.25 , and is then digitised. The 12-bit ADC has a built-in sample-and-hold amplifier, a full range of $10 \mathrm{~V}$ and a conversion time of $800 \mathrm{~ns}$. The ROE has two other flexibilities built-in.

- The full-well capacity of $\sim 120 \mathrm{Ke}^{-}$and the readout noise of $\sim 8 \mathrm{e}^{-} \mathrm{rms}$ (including analogue electronics contribution) means that the dynamic range of the CCD is undersampled by the ADC, which has a conversion of $\sim 30 \mathrm{e}^{-} / \mathrm{DN}$. To enable operation with resolution down to the readout noise, the first stage of the ROE analogue electronics has a selectable gain of $\times 1$ (default) or $\times 6$, selectable by a register bit loaded from the DPE using the LSL. The $\times 6$ range changes the conversion to $\sim 5 \mathrm{e}^{-} / \mathrm{DN}$, with an ADC full scale of $\sim 20 \mathrm{Ke}^{-}$.

- There is also a mode in which the amplifier filter time constants are increased by a factor 5 and the readout slowed down correspondingly to further reduce readout noise.

The ADC output goes to an 8192 word FIFO, which feeds the PISO and HSL circuitry. The FIFO eases timing requirements on the handshaking between the ROE and DPE.

\section{Testing}

Testing on the ground has been as follows.

\section{1. $C C D$ testing by e2v}

Testing carried out by e2v related to CCD manufacturing process tests and ensuring that the CCDs meet the requirements for the mission. Particular tests included:

- Manufacturing: temperature cycling, thermal shock, vibration, geometrical measurements.

- Performance: QE, dark current, amplifier responsivity

- Qualification: accelerated life, radiation.

\subsubsection{Radiation testing}

Radiation tolerance of CCDs is critical for space applications. Testing consisted of irradiating two devices from the flight batch with $\mathrm{Co}^{60}$ gamma rays at Brunel University's Centre for Radiation Damage Studies. $\mathrm{Co}^{60}$ gamma rays have a mean energy of $\sim 1.25 \mathrm{MeV}$ and cause predominantly ionising damage, 
although the energy is sufficiently high to produce some bulk damage (see e.g. Janesick 2001). The devices were tested before irradiation and after 10, 20 and $30 \mathrm{krad}(\mathrm{Si})$, corresponding to several years in orbit. The dose rate was $\sim 9 \mathrm{krad}(\mathrm{Si}) /$ hour, so that each radiation increment took $\sim 1$ hour.

Ionising radiation produces electron-hole (e-h) pairs in the CCD. For those generated in the insulating layers, the electrons are mobile and can diffuse away from the generation site, while the holes are left behind, building up a net positive charge, causing flatband voltage shifts. If the device is biassed, there is an electric field across many of the insulating layers which separates the e-h pairs, whereas the pairs are more likely to recombine if the device is unbiassed. For this reason, flatband shifts are $\sim$ four times greater if the device is biassed during irradiation, compared with an unbiassed device. For these tests the devices were biassed, in order to assess the worst case flatband shifts.

Bulk damage consists of traps in the band structure of the silicon between the valence and conduction bands and charge generation sites, created by displacement of atoms in the lattice. The traps have characteristic timescales for capturing and releasing electrons, which can lead to charge transfer efficiency (CTE) degradation. Charge generation sites can lead to "hot pixels".

Principal results were as follows.

- Flatband shifts: shifts of $125 \mathrm{mV}$ per $\mathrm{krad}(\mathrm{Si})$ were observed. This is in accordance with other results on $\mathrm{e} 2 \mathrm{v}$ devices (Robbins 2000).

- Dark current: for a typical substrate voltage, the dark current increased from $\sim 0.01 \mathrm{e}^{-} \mathrm{pix}^{-1} \mathrm{~s}^{-1}$ at $-85 \mathrm{C}$ to $\sim 0.04 \mathrm{e}^{-}$pix $^{-1} \mathrm{~s}^{-1}$ after $30 \mathrm{krad}(\mathrm{Si})$.

- CTE: CTE was measured using $\mathrm{Fe}^{55}$ X-ray events at $\sim-100$ C. Parallel CTE fell from $\sim 0.999995$ to $\sim 0.99996$. Readout register CTE was essentially unaffected.

- QE and Pixel Response Non-Uniformity (PRNU): these were also unaffected.

- Readout noise: this too was unaffected.

These results agree well with values reported in the literature (e.g. see Robbins 2000). In orbit, bulk damage will be caused by solar protons and could lead to a larger loss of CTE per $\mathrm{krad}(\mathrm{Si})$. The instrument structure provides shielding to reduce the dose.

\subsection{Testing at MSSL}

Testing at MSSL concentrated on the following factors.

- CCDs. Mapping of dark current, bright and dark defects.

- FPAe, ROE. Functional tests, workmanship inspections etc., interfacing to MSSL GSE (for ROE/FPAe/CCD control and data acquisition), interfacing to ESA-supplied DPE simulator (in conjunction with UCD/DIAS)

- CCDs with FPAe, ROE. Verification of correct imaging performance, responsivity and noise of CCD/FPAe/ROE combination. For example, Fig. 4 shows the results from a light transfer curve test used to measure the responsivity and noise. This test consists of measuring the signal and
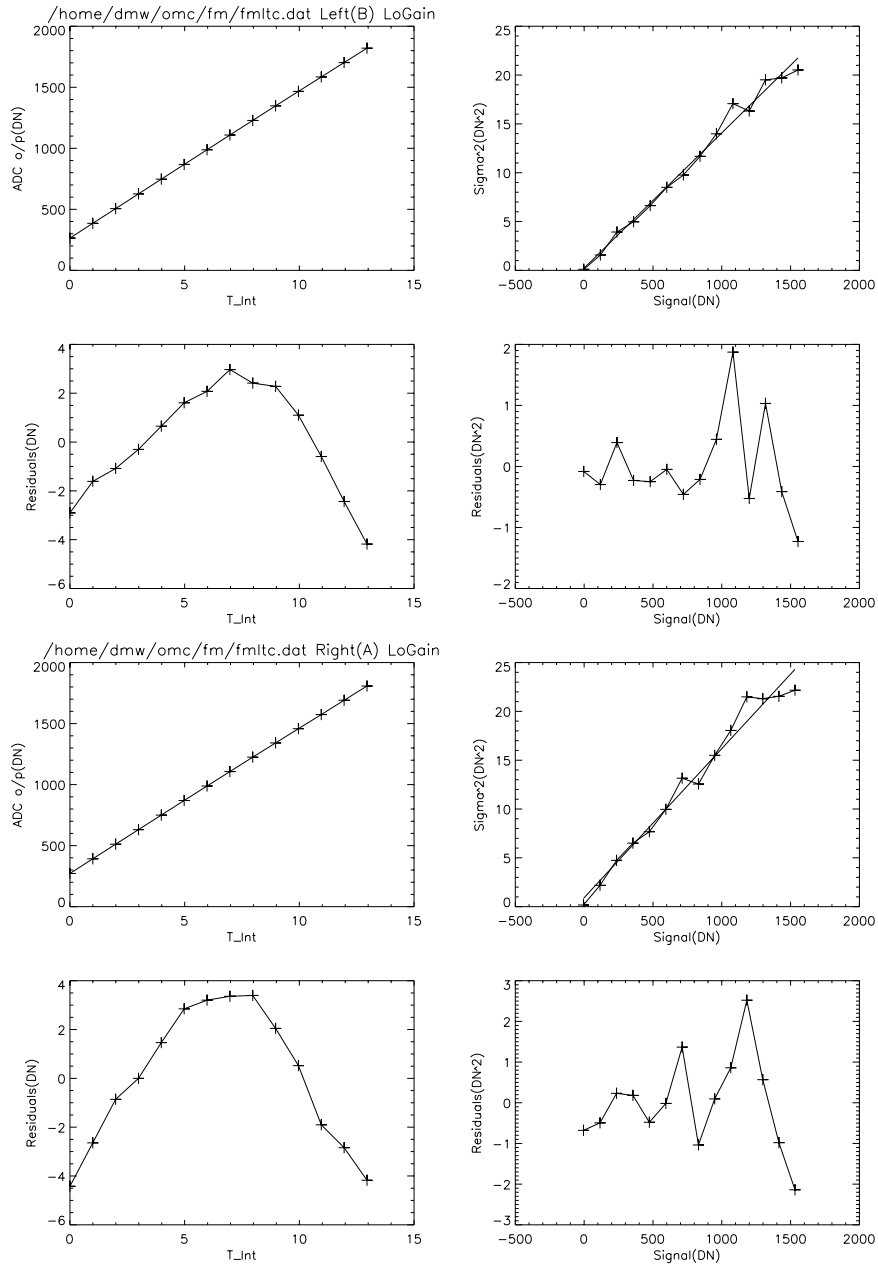

Fig. 4. Light transfer curve used to measure responsivity and readout noise.

noise over a range of signals from zero up to $\sim$ half fullwell. The top left plot shows the left channel ADC output (DN) as a function of integration time, while the plot below has the same horizontal scale and plots the residuals from a straight line fit. The top right plot shows noise squared $\left(\mathrm{DN}^{2}\right)$ against signal (DN): noise squared is the sum of the squares of readout noise and signal shot noise, so that this plot gives a straight line the slope of which is the system conversion factor, $\mathrm{DN} / \mathrm{e}^{-}$, and the intercept at zero signal gives the square of readout noise (for a detailed derivation see e.g. Janesick 2001). The plot below again shows the residuals. The bottom four plots follow the same sequence for the right channel.

\subsection{Instrument and S/C level tests}

Three full models were delivered to INTA:

- Engineering Model (EM).

- Qualification Model (QM), later refurbished as Flight Spare (FS).

- Flight Model (FM). 


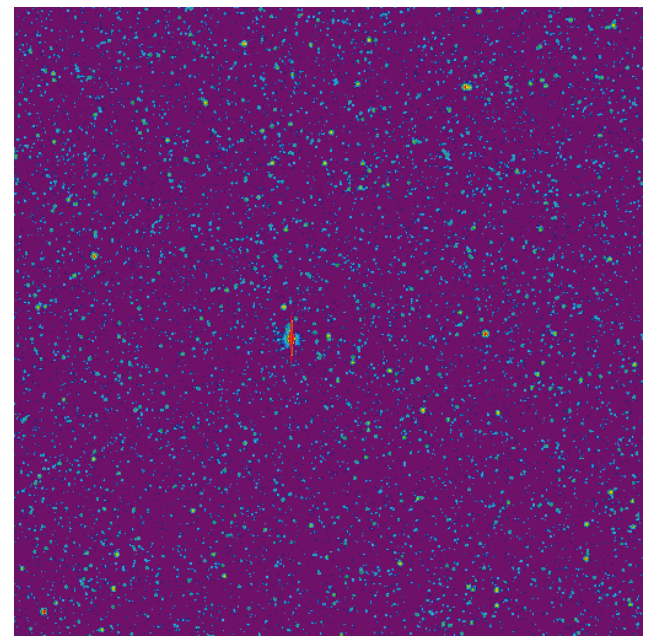

Fig. 5. $512 \times 512$ portion of OMC's first-light image, showing gamma Trianguli Australis.

At this level, tests included qualification and acceptance tests such as instrument vibration, and performance testing such as alignment, focusing of the FPA with the telescope and calibration, results of which are reported in Mas-Hesse et al. (2003).

\subsection{In-orbit results}

OMC was the first instrument on Integral to be commissioned. It has been operating successfully since October 2002 and has observed $>20000$ targets. Figure 5 shows one quarter $(512 \times 512$ pixels $)$ of the first light image in false colour.
An approximately logarithmic intensity function has been used so that the brightness indicates the stellar magnitudes rather than fluxes to give an indication of the density of stars visible. In this $10 \mathrm{~s}$ integration, a bright star near the centre saturates the CCD and causes blooming over several pixels. This is gamma Trianguli Australis, with visual magnitude 2.87.

OMC's strength is in acquiring light curves thanks to Integral's high orbit, so that long uninterrupted observations are possible. Thus OMC is particularly suited to observations of time-variable sources such as novae, eclipsing binaries and AGN. More detailed results are reported in Mas-Hesse et al. (2003), e.g. light curves of eclipsing binaries.

Acknowledgements. Funding for MSSL's development of the readout electronics and GSE was provided by the UK's Particle Physics and Astronomy Research Council (PPARC). Funding for the building of the EM, QM/FS and FM electronics and a portion of the CCD purchase was provided by UCD-DIAS through Prodex, with the remainder of the CCD purchase cost provided by INTA. We would also like to acknowledge the advice received from $\mathrm{e} 2 \mathrm{v}$ technologies and many helpful discussions. The authors thank the technical and administrative support staff at MSSL for their hard work throughout the project.

\section{References}

Janesick, J. R. 2001, Scientific Charge-Coupled Devices, SPIE monograph; PM83

Mas-Hesse, J. M., Gímenez, A., Culhane, J. L., et al. 2003, A\&A, 411, L261

Mazy, E., Defise, J. M., Plesseria, J. Y., et al. 2003, A\&A, 411, L269

Robbins, M. 2000, The Radiation Damage Performance of e2v technologies CCDs, e2v Technical Note S\&C 906/424 